\title{
Investigation of the Magnetic Interaction of Small Permalloy Particles
}

\author{
H. Koo, M. Dreyer, V. V. Metlushko, and R. D. Gomez, Member, IEEE
}

\begin{abstract}
Permalloy island arrays and undulated lines were systematically studied by MFM to clarify the effect of neighbor magnetic interaction. The islands are $860 \mathrm{~nm} \times 320 \mathrm{~nm}$ and $35 \mathrm{~nm}$ thick, single domains and patterned to have preferential interaction along the easy and hard axes. The switching field for easy axis interaction is relatively high, i.e., 370 Oe with a 32 Oe spread; while the hard axis interaction produces a switching field of 130 Oe and a slightly wider distribution of over 40 Oe. A third array is comprised of undulated lines with connected segments. Magnetic charges accumulate at the ends of each segment, and yield the strongest possible interaction along the easy axis. In this case all segments on one line simultaneously switch at a specific field. The switching field increases linearly from 150 Oe with the number of segments up to a maximum number of 50, and approaches a constant field of $290 \mathrm{Oe}$. The results favorably compare with the prediction for parallel rotation of the Jacobs-Bean chain of spheres model.
\end{abstract}

Index Terms-Array, chain of spheres model, Jacobs-Bean model, magnetic interaction, parallel rotation, permalloy, switching distribution, undulated line.

\section{INTRODUCTION}

A $\mathrm{N}$ IMPORTANT consideration in the applications being conceived for small patterned elements such as high density recording media, microsensors, logic devices, is the influence of neighboring islands on the magnetic properties of the individual islands. The effect is not yet well understood, and one can find diverging conclusions regarding the effects on reversal field with particle interactions [1], [2]. In principle, the problems appear simple in that the neighboring elements produce stray fields that cause the neighboring particles to behave differently from the isolated case. However, vastly different values for the reversal switching fields can be obtained depending upon the models used for the reversal mechanism. In this work, we attempt to clarify the situation by systematically observing the reversal of single domain permalloy particles with different particle interactions using magnetic force microscopy, and comparing the theory of Jacobs-Bean chain of spheres model in the extreme case of maximally coupled magnetic elements.

Manuscript received October 13, 2000; revised February 7, 2001.

This work was supported in part by NSF CAREER AWARD ECS-9984797

H. Koo, M. Dreyer, and R. D. Gomez are with the Department of Electrical and Computer Engineering, University of Maryland, College Park, MD 20742 USA. They are also with the Laboratory for Physical Sciences, College Park, MD 20740 USA (e-mail: hkoo@eng.umd.edu).

V. V. Metlushko is with the Department of Electrical Engineering and Computer Science, University of Illinois, Chicago, IL 60607 USA.

Publisher Item Identifier S 0018-9464(01)07278-8.
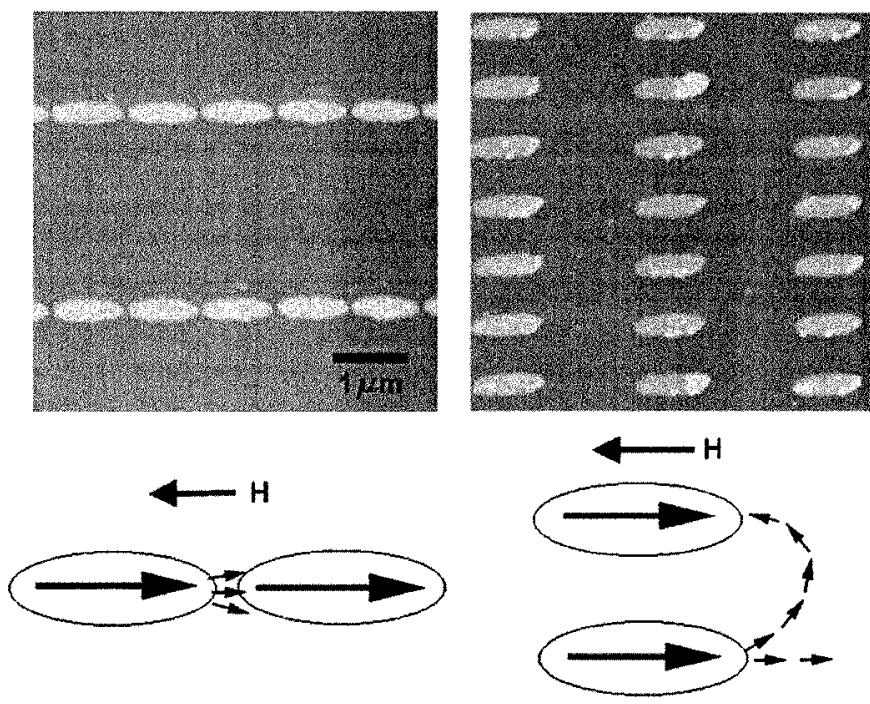

(a)

(b)

Fig. 1. Topography and schematic diagram of magnetic interactions along (a) the easy axis and (b) the hard axis.

\section{EXPERIMENTAL RESULTS AND ANALYSIS}

Three types of island arrays were fabricated which are classified in the following way. Array A particles interact along the easy axis, array $B$ particles weakly interact along the hard axis, and array $C$ is an undulated line strip where each segment is in contact with its nearest neighbors. The islands were deposited on Si substrates with native oxides using thermal evaporation and patterned using e-beam lithography and lift-off. The size of the individual island is nominally $860 \mathrm{~nm} \times 320 \mathrm{~nm}$ and $35 \mathrm{~nm}$ thick.

The topography of arrays $A$ and $B$ obtained using AFM is shown in Fig. 1. In array $A$, the distances between the islands along the easy axis and the hard axis are $70 \mathrm{~nm}$ and $2500 \mathrm{~nm}$, respectively, so that the interaction is mainly along the easy axis. In array $B$, the distances between the islands are $1500 \mathrm{~nm}$ and $570 \mathrm{~nm}$ along the easy and hard axes, respectively, so that the interaction, albeit weak, is along the hard axis. The arrays were placed in a magnetic force microscope with a variable external field [3], and successive magnetic images of the islands were obtained as the applied field was first increased to $+500 \mathrm{Oe}$ and gradually decreased to -500 Oe in increments of 10 Oe. The effective external field is combination of applied field and stray field from the tip, but tip effect is very weak compared to reversal field. 


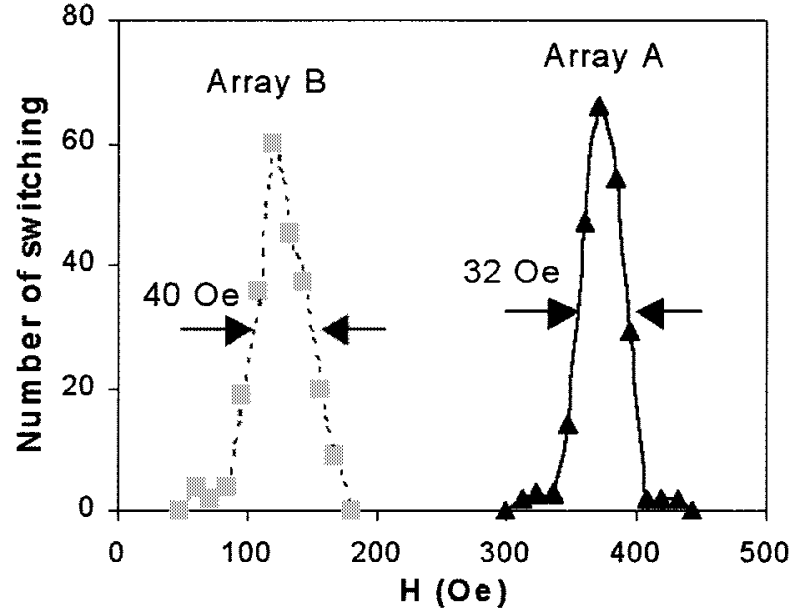

Fig. 2. Switching distribution curves of array $A$ and $B$. The interactions predominate either along the easy axis (array $A$ ) or along the hard axis (array $B)$

The switching field distributions are shown in Fig. 2, showing the striking effect of the interaction. The peak of switching curve is 370 Oe for array $A$, which is nearly three times that of array $B$ at 130 Oe. In addition, array $A$ has a sharper distribution than $B$. The FWHM is 32 Oe for array $A$ in contrast with 40 Oe for array $B$. The schematic diagram shown in Fig. 1(a) helps to understand the interaction in array $A$. In the initial state, i.e., at remanence, the moments are aligned in the same direction. In this configuration, the stray fields from the nearest neighbors are mainly parallel to the magnetization direction of an island thereby stabilizing the moment. The magnitude of the stray field can be estimated by using a monopolar source and a calculating azimuthal component at the position of the nearest neighbor. Based on the material and geometry of our samples, we estimate this field to be about 156 Oe at the edge. As shown in Fig. 1(b) the interaction field in array $B$ is anti-parallel to the moment and thus causes a net reduction in the switching field. However, the magnitude of the interaction field is only about $0.92 \mathrm{Oe}$, which is too weak to produce a significant departure from the switching field of isolated islands. Using micro-magnetic simulations, a switching field of 140 Oe was obtained for the islands whose dimension and arrangement are same as array $B$. This value is very similar to that found for isolated island of the same geometry. Other researchers, using closely spaced island arrays have found significant reduction in the switching field for hard axis interaction [1].

Our results, however, appear to be opposite to the observations of Gibson and Shultz [2], where they observed that the switching field is lower for easy-axis interacting islands than isolated islands. The reduction is attributed to a so-called, "trigger-mechanism" where the islands of lower switching field promote the reversal of neighbors. A possible explanation to reconcile our results with theirs is by considering the differences in the island geometry. Their islands have 10:1 aspect ratio and are about $100 \mathrm{~nm}$ wide, and the reversal process occurs through end domain nucleation and propagation. The high aspect ratio produces stray field that rapidly decay with distance, and hence the interaction in those islands is confined at the domain ends. Hence, it is highly likely that a reversed

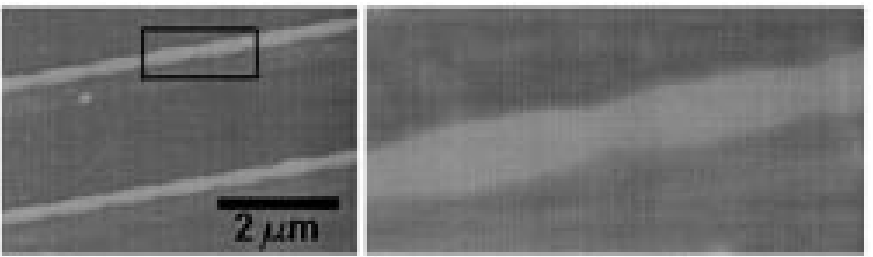

Fig. 3. Topography of array $C$. The lines are consists of connected segments.
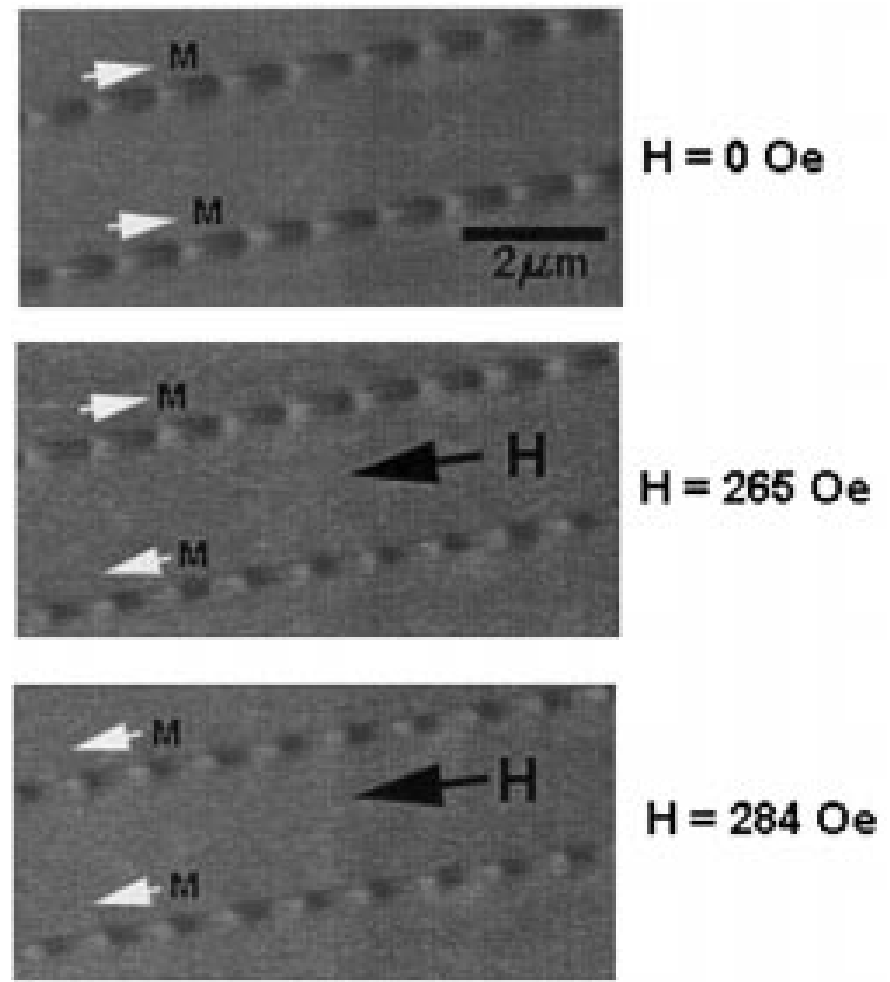

Fig. 4. Magnetic reversal process of undulated lines. All segments on the same line flip at the same time.

magnetized neighbor preferentially promotes the formation of reversed end domains and causes the "trigger effect."

In our islands, the effect of the "trigger mechanism" is to reduce the switching field distribution. A reversed neighbor induces a field in the same direction as the applied field. Thus, once a neighbor is switched, a smaller external field is needed to reverse the island. The reduction of nearly $10 \mathrm{Oe}$ in the switching distribution for array $A$ compared with array $B$ supports this conclusion.

Following this reasoning, we therefore expect that in the limit of very strong easy axis interaction, the spread of the switching field distribution should approach zero. We explore this assertion by fabricating arrays with the strongest possible interaction along the easy axis. We prepared continuous strips, with periodic undulations of the width. The topography of array $C$ scanned using AFM is shown in Fig. 3.

The periodic constrictions produce variations in the local magnetization, so that magnetically, the strip becomes a row of interconnected single domain segments. The MFM image in Fig. 4 shows the bipolar accumulation of magnetic charges for each segment. The pattern is the extreme case of array $A$, with the maximum nearest neighbor interaction. The top image 


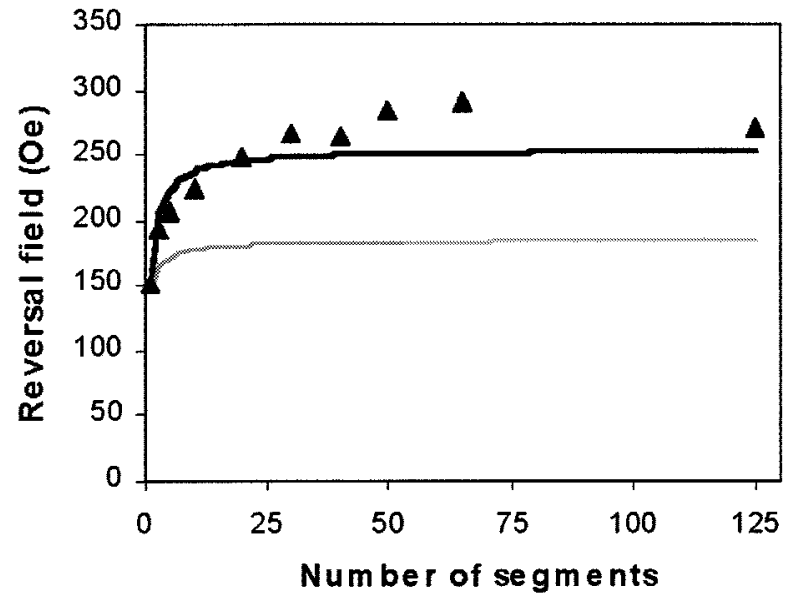

Fig. 5. Reversal field as a function of the number of segments. This graph shows experimental data (markers), parallel rotation model (heavy solid line) and fanning model (light solid line).

of Fig. 4 is the magnetic structure without field, while middle and bottom images show the reversal of various rows with increasing applied field. At $H=265$ Oe the magnetization direction of the lower line is reversed, while a field of 284 Oe, similarly reverses the upper row. An important property of these island arrays is that all segments in a given row switch at the same time. In other words, the spread of the switching field distribution $\left(\Delta H_{s w}\right)$ is zero. Indeed the interaction of undulated line does not appear to further increase the reversal field but affects the spread of distribution. The fact that the island array $A$ apparently switches at a higher field is due primarily to the larger aspect ratio of the islands in array $A$ than the individual segments in the undulated line. Having established that $\Delta H_{s w}$ is zero for a given row, we now address the problem of how the length of each row determines its switching field. To this end, we systematically cut the lines into several segments, covering a range of 1 to 125 segments. We used contact mode atomic force microscopy with very stiff cantilevers to scrape material and form gaps of about $500 \mathrm{~nm}$ wide. We then performed the usual MFM switching experiments on each of these line segments.

Fig. 5 shows a summary of the results. The reversal field is plotted as a function of length, i.e., the number of segments. The data shows that the switching field increases with the number of segment from 150 Oe for a single element and up to about 290 Oe for 50 segments. The switching field approaches constant value if the number of segments is over 50 .

To interpret the data, we are aided by Jacobs and Bean's chain of sphere model [4], which predicts specific functional dependence of the switching field with number of segments. The basic assumption of chain of sphere model is magnetic dipole-dipole interaction. The model predicts two possible mechanisms, namely the parallel rotation and the fanning mode. In the former case the moments rotate in parallel unison, while in the latter case the moments rotate in alternate orientations. The original model assumes perfectly spherical elements and calculates the coercivity due to the magnetic interaction of islands without the affects of shape anisotropy. In order to calculate the total coercive force of the undulated line, we slightly modify the Jacobs and Bean's model to include the intrinsic coercivity of single segment, $H_{k}$. The intrinsic coercivity is a function of demagnetization factor of segment and depends on the particle dimensions [5]. Furthermore, in interpreting the model, we assume that dipole moment $\mu$ is the saturation magnetization of permalloy times the volume for each segment, $n$ is the number of segments, and the distance, $a$, between the dipoles is the wavelength of the undulation. Thus total coercivity for the parallel rotation model can be expressed as,

$$
H_{c, \text { total }}=6 \frac{\mu}{a^{3}} \sum_{j=1}^{n} \frac{(n-j)}{n j^{3}}+H_{k} .
$$

This model does not include the factor of crystalline anisotropy as it is negligible for permalloy. In the case of materials with strong crystalline anisotropy such as cobalt, the calculation of $H_{k}$ will be more complex. The expression for the fanning model is similar to (1) except that it contains a term that accounts for the angular divergence of the moments and thus reduces the coercive field. In parallel rotation model, exchange energy is constant because magnetic moments of all segments are always parallel. To get the coercive field we let the derivative of the total energy respect to angle with easy axis equals to zero. However, derivative of exchange energy is zero, so this term can be neglected to calculate coercive field. In fanning model, Jacobs and Bean assume point contact, so it may not explain the switching mechanism of the undulated lines.

The predictions of the Jacobs and Bean's parallel rotation model as well as the results for the fanning model, are plotted in Fig. 5. It is to be emphasized that there are no adjustable parameters in the models. Notwithstanding the uncertainty in the data, the prediction of the parallel rotation model is closer to the experimental results, which suggests that parallel rotation is the mechanism of magnetization reversal. In fact, high resolution MFM images show that the magnetic moment of each segment makes the same angle with the easy axis just prior to switching process.

\section{REFERENCES}

[1] M. S. Wei and S. Y. Chou, "Size effects on switching field of isolated and interactive arrays of nanoscale single-domain Ni bars fabricated using electron-beam nanolithography," J. Appl. Phys., vol. 76, pp. 6679-6681, 1994.

[2] G. A. Gibson and S. Shultz, "Magnetic force microscopy study of the micromagnetics of submicrometer magnetic particles," J. Appl. Phys., vol. 73, pp. 4518-4521, 1993.

[3] H. Koo, T. V. Luu, and R. D. Gomez, "Slow magnetization dynamics of small permalloy islands," J. Appl. Phys., vol. 87, pp. 5114-5116, 2000.

[4] I. S. Jacobs and C. P. Bean, "An approach to elongated fine-particle magnets," Phys. Rev., vol. 100, pp. 1060-1067, 1955.

[5] A. Aharoni, "Demagnetizing factors for rectangular ferromagnetic prisms," J. Appl. Phys., vol. 83, pp. 3432-3434, 1998. 